\title{
EXPERIMENTOS CUALITATIVOS. UNA FORMA DE ABORDAR EL ELECTROMAGNETISMO
}

\section{Luis Miguel Beltrán Sierra - luim.beltran@unisabana.edu.co* José González Flórez - gonzalej @ uni.pedagogica.edu.co**}

\begin{abstract}
In this work a different way to approach the electricity and magnetism course for first level university students is discussed, specially within programs for teachers education. It suggests two aspects: a historical-epistemological review of Faraday's work and the realization of a series of experiments which will allow students to grasp a more meaningful development of electricity and magnetism. We hope this two aspects will be worked in the classroom as "problematical situations"(Garret 1988, Pozo 1994) to allow students to be involved in problems solutions and get closer to "scientific work" and particularly to the physicists "doings" (Flórez, 1994).
\end{abstract}

\section{RESUMEN}

En este trabajo se propone una forma distinta de abordar el curso de electromagnetismo para los primeros niveles de universidad y especialmente para formación o capacitación de docentes. Sugiere dos aspectos: Una revisión histórico-epistemológica de la obra de Faraday y la realización de una serie de experimentos que le permitirán a los estudiantes ver con más sentido el desarrollo del electromagnetismo. Se espera que estos dos aspectos sean trabajados en el aula de clase como "situaciones problema" (Garret 1988, Pozo 1994); que permitan involucrar a los estudiantes en la solución de problemas y de esta manera acercarse a la forma del "quehacer científico" y particularmente al "hacer"de los físicos (Flórez, 1994).

Palabras claves: Historia, epistemología, Faraday, situaciones problema, experimentos.

\section{ENSEÑANZA SUPERFICIAL DE LAS CIENCIAS}

Casi que paralelamente al desarrollo de los esquemas explicativos del universo, han ido surgiendo escuelas psicológicas que intentan describir los procesos de desarrollo cognitivo del individuo. Bajo estos enfoques se han montado sistemas de enseñanza que pretenden ceñirse a las sugerencias o postulados de dichas escuelas. En este tipo de proceso se ha visto envuelta obviamente la enseñanza de las ciencias y consecuentemente la utilización del experimento como elemento pedagógico (Hodson, 1988).

Respecto a este tema, es bastante lo que se ha reflexionado, sin embargo a pesar de su carácter de reflexión, muy pocos recogen una decidida o concisa propuesta, dirigida

\footnotetext{
*Profesor Facultad de Ingeniería Universidad de la Sabana. Bogotá D.C. Colombia.

${ }^{\star *}$ Profesor Departamento de Física. Universidad Pedagógica Nacional. Bogotá D C. Colombia.
} 
hacia los maestros, con respecto al enfoque que pudieran dar a tales experimentos (Furió y Gil, 1989; Gil 1991).

Se sabe de la importancia que ha tenido el experimento para el desarrollo de la ciencia, haya sido ideado éste, para buscar una conciliación de una particular visión del universo, caso en el cual no necesariamente es cuantitativo, o para mediar entre teorías en pugna. Cualquiera que sea el caso, se sabe que un experimento no se monta porque si, sin tener un objetivo claro, definido y una estructura conceptual preconcebida. Así, al menos que el experimento no esté dentro del contexto de una visión o metafísica particular o una teoría ya estructurada, carece de todo sentido (Berkson, 1981; Papen, 1982).

Cuando se trata de la enseñanza de una ciencia aunque hay un cambio radical de contexto, el experimento no deja de ser menos importante a pesar de la gran diferencia en cuanto a su objetivo. Al respecto, la experiencia ha mostrado que dicha enseñanza falla desde el punto de vista de la utilización del experimento como elemento pedagógico. Una de las causas de esa falla, podría atribuirse a la pobre o falsa concepción de ciencia que tenemos los maestros y a la resistencia a reflexionar sobre ello. Resulta más cómodo mantenerse en un esquema tradicional de enseñanza, entrando en una rutina que hace olvidar de la responsabilidad que tiene el maestro, sobre todo si de formar educadores se trata.

Por lo general se pretende que nuestros estudiantes reciban -no reflexionen esta ciencia, reduciendo el constructo matemático al producto final de las teorías, que se presenta a manera de resumen en los libros o textos. Este método, más la cómoda mirada a tal tipo de presentación, obviamente limitan cualquier visión que se pueda esperar de la ciencia y promueven una falsa concepción de la misma. Una vez que son re-deducidas las ecuaciones (Hammer 1994) que incluso algunos llamamos irresponsablemente, fórmulas- que sustentan matemáticamente, por así decir a la teoría, se pasa a adiestrar en su aplicación en la solución de ejercicios y problemas que casi siempre son hipotéticos (Colombo y Fontdevila, 1990).

Con respecto al tipo de texto mencionado, se deben revisar los propósitos de su uso como guías o texto base. Pues con ellos, lo mejor que se puede obtener al final de un determinado programa, son algunos buenos solucionadores de ejercicios, problemas hipotéticos y rebuscadores de 'fórmulas". Eso puede estar bien para algunos, pero y ¿dónde está la ciencia? Formar un estudiante bajo esta perspectiva, es hacerle creer que la ciencia es una estructura cuyo objetivo es crear aplicaciones, hacerle cómoda la vida al hombre y no desvelar el misterio del universo (Gil, 1983). Esto quizás puede ser válido si lo que estamos formando son ingenieros, pero no físicos o maestros de física.

Otro enfoque es presentar la teoría como una verdad acabada, quizá porque nuestra falta de reflexión hace que con el tiempo, nosotros mismos así lo creamos, se olvida hacer énfasis a los alumnos, de que se trata justamente de teorías, y que por tanto son estructuras o modelos aceptados por cuanto tienen temporalmente la capacidad de explicar fenómenos que parecen dar cuenta del comportamiento del universo. De otra forma se da al estudiante la imagen de que todo ya está hecho y terminado y que, por ende, nada hay para discutir ni reflexionar. Uno de los problemas de la enseñanza de la ciencia es justamente que se transmite a los alumnos, un sentimiento de aceptación absoluta de las explicaciones y hacemos que las consideren como verdades últimas y acabadas (Papert, 1982). 
El error está en suponer que el alumno adquiere un verdadero conocimiento cuando éste es simplemente transmitido o dado (Paped, 1995). Proporcionarles las explicaciones, contribuye a la dependencia, a eliminar la necesidad de pensar por sí mismo y a generar impotencia intelectual en el individuo. Este hábito de dependencia y la sensación de impotencia, hacen que el estudiante una vez absorbido por el sistema de enseñanza, se resista a cambiar su actitud frente al conocimiento. Es posible que creamos que al proporcionar la mayor cantidad de explicaciones, el proceso intelectual del estudiante se facilite y mejore, pero de hecho sucede todo lo contrario, se hace más superficial y de menor calidad. Un maestro que recurra a dar explicaciones y demostraciones, está suponiendo que él, ya conoce lo que el estudiante necesita (Meneses y Caballero, 1995).

Una significativa enseñanza de la ciencia debe apuntar hacia la percepción y comprensión de lo esencial ${ }^{1}$, antes de intentar rededucir estructuras matemáticas, sólo cuando lo esencial sea percibido, debe buscar la forma de orientar hacia los elementos que permitan ampliar la percepción de sus alumnos. Esta tendencia natural o adquirida a percibir lo esencial de los asuntos científicos es lo que debe distinguir al verdadero aprendizaje de las ciencias. Los alumnos a los que no se les brinda la oportunidad de conocer lo esencial, se van creyendo conocer lo que realmente es importante. Para ellos será difícil distinguir y entender lo que es superficial, pues no tuvieron opción de explorar lo que es profundo.

Renunciando a esta manera de enseñanza, se queda ante el problema de encontrar cuestiones a las que el estudiante pueda hacer frente cómodamente con lo que tiene, de forma que a partir de allí, sus competencias intelectuales puedan ser liberadas y desarrolladas.

\section{ALTERNATIVA DE ENSEÑANZA}

Todo esto nos lanza a pensar en otra sugerencia. El objetivo primordial de un estudiante de física o aspirante a maestro de física (con mayor razón), no debe ser adiestrarse en la solución de ejercicios, sino en el análisis con sentido crítico de las teorías. Para esto es de gran importancia, que se inmiscuya en sus antecedentes, que busque su génesis, que siga con detalle el proceso de desarrollo, que analice los elementos internos y externos que contribuyeron en su construcción (Furió y Guisasola, 1997). Seguramente que se ganaría mucho en comprensión, análisis e incluso en reflexiones de validez, de tipo filosófico o epistemológiço hacia la ciencia, despertando mayor interés e incluso sentimientos de admiración, respeto y deseo de imitar o seguir el camino de los hombres que han contribuido siempre a forjar la ciencia. El principal objetivo de estos estudiantes o los de ciencias en general, debería ser, poder llegar a participar y contribuir en el proceso científico de su época (Papert, 1995).

Ahora bien, una propuesta concreta es intentar inducir al estudiante en este tipo de formación a través de la reflexión sobre ciertos fenómenos que se puedan reproducir en el laboratorio con algunos experimentos.

Sin entrar en una discusión sobre las teorías del conocimiento, reconocemos que es cierto, que de alguna manera es conveniente, que el individuo sea constructor de su conocimiento. Desde este punto de vista, la propuesta apunta hacia la utilización del

\footnotetext{
${ }^{1}$ No se tome como connotación de básico o elemental. sino como de esencia o profundidad, que para nosotros lo constituirla e verdadero y completo análisis de una teoría.
} 
experimento como elemento de motivación y reflexión alrededor del cual, se generen necesidades de explicación y que como consecuencia, involucra y encauza al estudiante hacia la investigación y análisis de los esquemas explicativos primarios (Chaparro et al, 1997), su evolución y sus criterios de validez. Así, esta reflexión debe conducir a discutir por qué la explicación de un fenómeno se toma como válida a la luz de un cierto esquema y no de otro, o por qué un hecho o experimento en particular suscita un cambio radical de concepción acerca del universo.

Consideramos que con esto, el estudiante no sólo se inmiscuye en el verdadero análisis de las teorías -objetivo primordial- sino que se va involucrando a su vez, paulatinamente con mayor profundidad en las concepciones e imágenes de ciencia, ganando seguridad para emitir juicios de tipo crítico. Con este proceso, los constructos matemáticos llegan como elementos que forman parte de ese análisis de las teorías y no como los "primeros planos" de las mismas.

Ahora bien, si se tiene la intención de utilizar el experimento como elemento constructor de conocimiento, serian válidas las siguientes preguntas: ¿Qué fenómenos convienen presentar al estudiante? y ¿cómo dirigir el experimento o análisis del fenómeno para que pueda cumplir el objetivo de ser constructor?

Respecto a la primera, el profesor Bautista (1990) sugiere: Para seleccionar los fenómenos presentados en el aula, se debe pensaren aquéllos que sean fundamentales de acuerdo al desarrollo que se esté dando a una teoría. Es decir, 'los fenómenos cuyo tratamiento sea importante para evidenciar la necesidad de un cambio de concepción o construcción de una nueva teoría y una nueva imagen del mundo: Fenómenos que resulten cruciales desde el punto de vista didáctico ${ }^{2}$. Si se logra que el estudiante los interprete y vea la necesidad de una nueva teoría, se habrá logrado un avance significativo".

La propuesta toma como base este tipo de reflexión y motiva a hacer un trabajo que implica la búsqueda de algunos de esos fenómenos cruciales, el diseño de los experimentos y algunas sugerencias de tipo pedagógico para presentarlas a manera de ejemplo en este artículo.

En cuanto a la segunda pregunta, es difícil dar una respuesta concreta, por cuanto depende, respecto del maestro, de su habilidad, creatividad, capacidad, didáctica y algún tipo de conocimiento sobre el desarrollo cognitivo del alumno.

Por parte del alumno, claridad en Los conceptos involucrados, una buena capacidad de análisis, suspicacia, inconformismo e interés (Beltrán,] 996).

\section{CONSTRUCCIÓN DE LA TEORÍA DE CAMPOS}

WI ejemplo que aquí se desarrolla, está aterrizado en un tema que se podría resumir en la pregunta ¿Qué estrategias utilizar para que el estudiante logre una buena y sólida concepción de la teoría de campos? (Funió y Guisasola, 1997). Cuestionamiento que a su vez surge del hecho de que se detecta, tanto en el ámbito medio como superior, una gran dificultad para lograr una buena comprensión de dicha teoría (Furió y Guisasola, 1998).

\footnotetext{
${ }^{2}$ Este tipo de experimento, es más enriquecedor y motivante, pues exige una reflexión más profunda, hace ver que las explicaciones no son verdades absolutas y que pueden ser transitorias.
} 
Dada su extensión se considera necesario tomar una parte específica del desarrollo de la teoría. (Hilborn 1998). Para nuestro caso, tomaremos la transición del concepto de estado electrotónico al de líneas de fuerza en la obra de Michael Faraday (Faraday).

Identificado el problema, una alternativa es basarnos en la utilización de experimentos claves y dirigirlos de manera adecuada para involucrar al estudiante en el razonamiento e investigación del por qué la necesidad de un nuevo esquema explicativo y qué validez puede tener. En e caso particular de la construcción de la teoría de campos como un todo, un proceso basado en experimentos pedagógicos, debe darse lentamente y por etapas, que podríamos sugerir así:

1. Análisis de fenómenos electrostáticos.

2. Análisis de fenómenos magnéticos

3. Acciones entre corrientes.

4. Acciones corriente - imán (Experimento de Oersted) (Gómez,] 998).

5. Experimentos de Faraday sobre la inducción de corrientes por movimiento de imanes y bobinas.

6. Análisis de Maxwell - determinación de la velocidad de las ondas electromagnéticas.

7. Experimentos de Hertz.

La presentación y manejo pedagógico de los experimentos en cada etapa, debe hacerse buscando una relación can la siguiente. Tal vez no se ha dicho explícitamente, pero es de suponer que si lo que perseguimos es el análisis de por qué la necesidad de un cambio de explicación, el estudiante ya tiene un esquema explicativo del mundo (concretamente con relación a la acción entre los cuerpos). Generalmente cuando se llega a este punto de la teoría del campo electromagnético, dicho esquema es el Newtoniano (Berkson, 1981; Furió y Guisasola, 1998).

Otra cosa importante a destacar es, que el alumno va dando las pautas para seguir a la siguiente etapa, donde otra Serie de experimentos debe prepararse, no es el maestro quien tradicionalmente llega al aula o laboratorio a decir "hoy vamos a ver....etc., etc.".

Supongamos ahora que se intenta analizar de dónde y cómo surge el concepto de líneas de fuerza. Antes de traer a discusión, los experimentos que hemos denominado claves, pensamos que es conveniente que el estudiante, previamente haya sido inducido dentro de un proceso de razonamiento dirigido a través de la observación de otros experimentos que en un principio no cuestionen demasiado su esquema newtoniano, pero que poco a poco introduzcan elementos que le creen situaciones de conflicto (White, 1993), Podrían ser por ejemplo:

- Interacción (atracción - repulsión) entre cuerpos cargados electrostáticamente

-- Interacción (atracción- repulsión) entre imanes

- Experimento de Oersted (variación de elementos actuantes y tipo de acción)

- Experimentos de Ampére de interacción entre alambres con corriente, bobinas con corriente y éstos con imanes

- Las nuevas observaciones de Faraday con respecto al experimento de Oersted

— Las rotaciones electromagnéticas

- El electroimán

- La inducción electromagnética (hasta el momento de ser concebida como efecto o variación del estado electrotónico). 
Buscando en cada uno de ellos, el objetivo de involucrar al estudiante en la reflexión, la investigación y el análisis de las explicaciones.

\section{PROPUESTA DEL TRABAJO EXPERIMENTAL}

Para nuestro objetivo particular se proponen los siguientes experimentos ${ }^{3}$ :

\section{Experimento 1: Interacción extraña entre imanes}

Antes de este experimento y como se sugirió más arriba, el estudiante debió haber observado la interacción (atracción repulsión) lineal entre los imanes (Figura 1). Estas observaciones pueden ser aceptadas sin problema en un esquema newtoniano, pues resultan ser acciones centrales y entre cuerpos de la misma naturaleza (cuerpo magnético con cuerpo magnético).

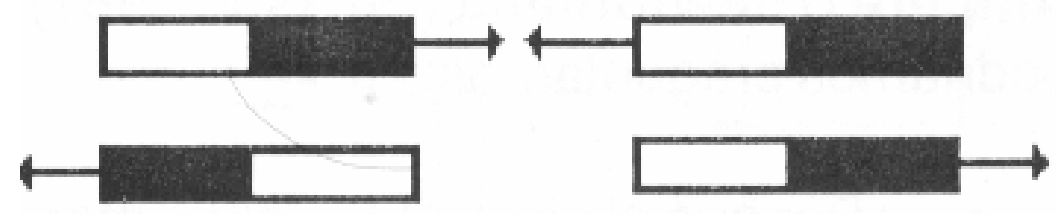

\section{Figura 1}

Ahora, se colocan en piar tos paralelos, un imán de barra Ay una aguja magnética a un imán $B$ de menor masa que el anterior (la idea es que no tenga mucha inercia), de modo que $B$ permanezca sobre una superficie y tenga un punto de rotación. Sobre éste, se suspende el imán $A$, tal que tenga la posibilidad de ser rotado (Figura 2).

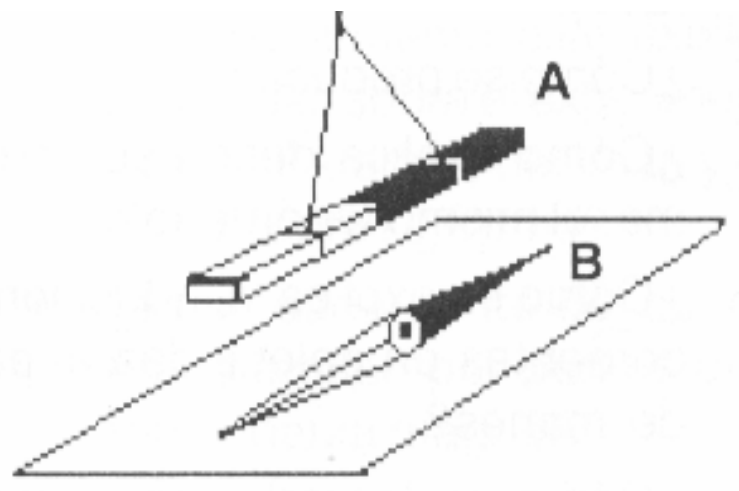

Figura 2

La distancia entre los imanes debe ser tal que ningún efecto de atracción-repulsión se manifieste cuando los imanes estén en reposo.

Al rotar el imán A, el imán B lo hace también. Puede parecer simple, pues ya vieron que la interacción entre cuerpos de la misma naturaleza nada tiene de extraño. Sin

\footnotetext{
${ }^{3}$ El maestro podría introducir otros si lo considera conveniente. El diseño, construcción del equipo y su montaje de los que aquí se presentan, se encuentra en el departamento de física de la U.P.N.
} 
embargo si se llama la atención para que se analice con más calma, se verá que la situación ya no es tan simple como una acción de tipo directo o mejor central. Preguntas que podrían generar la reflexión e investigación serían:

- ¿Qué dirección debe tener una fuerza sobre un cuerpo, para que su efecto sea la rotación del mismo?

-- ¿Se puede explicar el efecto observado mediante el concepto de fuerza central?

- ¿Qué implicaciones tiene la observación de una fuerza no central en el esquema newtoniano?

- ¿Qué explicaciones surgieron en la época del descubrimiento?

— ¿Cuáles puede considerar lógicamente válidas y por qué?

\section{Experimento 2: Acción entre cuerpo magnético (imán) y otro no magnético}

Se torna un disco (Puede ser de aluminio o de cobre) de unos $10 \mathrm{~cm}$ de radio y de $1 \mathrm{a}$ $2 \mathrm{~mm}$ de espesor, con la posibilidad que se pueda rotar Sobre éste, a una distancia tal que no haya atracción y en un plano paralelo al disco, se suspende un imán (Figura 3).

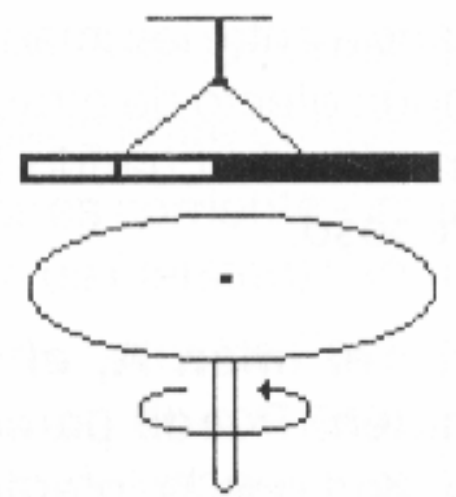

Figura 3

\section{Aquí, se recomiendan las siguientes etapas:}

1. Con un disco de madera o acrílico, donde no se observará ninguna acción al ser rotado, se descarta la posibilidad de que ese movimiento de rotación pueda generar corrientes de aire que hicieran posible el movimiento del imán.

2. Con un disco ferromagnético (lámina de hierro o acerada). Al rotar el disco el imán empieza a rotar.

- ¿Cómo dos cuerpos de diferente naturaleza (magnético y no magnética) pueden interactuar? ¿Es concebible en un esquema Newtoniano?

- ¿Qué tipo de acción está experimentando el imán para que su efecto sea de rotación?

- ¿De dónde proviene esa acción? 
- ¿Se vuelve el disco un imán con sólo rotario?

- ¿Lo permitiría el material de que está hecho?

- ¿Cómo lo comprobaría?

- ¿A quién podría atribuirse un primer esquema explicativo?

- ¿Es razonable el fenómeno desde un punto de vista Newtoniano?

3. Con un disco de aluminio o cobre, se observa el mismo efecto de rotación del imán, una vez el disco empieza a rotar (es una situación que resulta sorprendente). Aparte de suscitar las mismas preguntas que en el caso anterior, aquí se puede aprovechar la ocasión para inducir al estudiante a investigar y sopesan las explicaciones de Ampére y Faraday.

Si se sirnpatiza por la visión de Arnpére o newtoniana (Berkson 1981), podríamos preguntarnos:

- ¿Porqué el disco "se vuelve" magnético sólo cuando nota?

- ¿Si lo que se tiene es Lina acción entre corrientes, qué es una corriente para Ampére?

- ¿Es consistente su teoría de la corriente?

- ¿Por qué se manifiesta tal corriente sólo cuando el disco rata?

Si nos inclinamos por la visión de Faraday (Berkson 1981):

- ¿Qué es una corriente para Faraday?

- ¿Cómo se produce?

- ¿Cómo explica desde su esquema, el mismo fenómeno?

- ¿Cómo se explica la inducción de corrientes en solenoides al paso de imanes?

- ¿Cómo se lograría, observando estos experimentos, obtener una corriente 120 permanente?

\section{Experimento 3: Corrientes permanentes a expensas del magnetismo}

Se hace rotar el disco de cobre entre polos diferentes de un imán y se conectan dos cables a un galvanómetro sensible (ojalá menos de 30 microamperios), cuyos extremos pueden darse al estudiante para que intente detectar las corrientes que supuestamente se inducen (Figura 4)

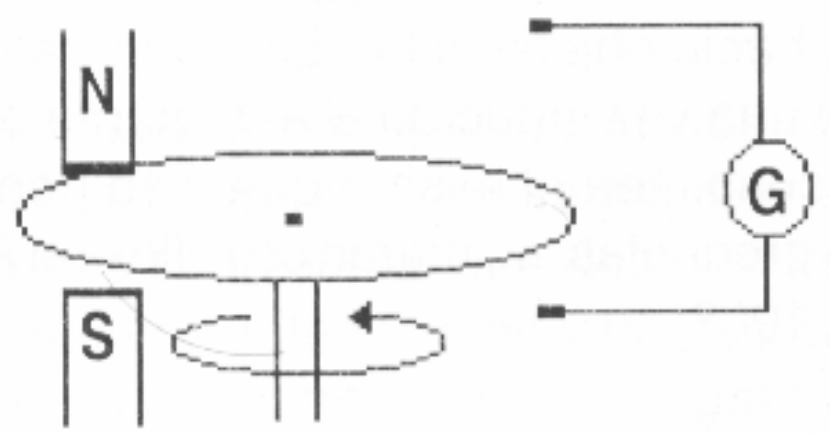

Figura 4 
Podríamos preguntarnos aquí:

- ¿Cómo se explicaría este hecho desde la perspectiva de Ampére?

- ¿Puede hacerlo Faraday desde su perspectiva?

- ¿Cuál es la diferencia con respecto a las experiencias con imanes y bobinas?

- ¿Afectan las conclusiones sobre este experimento, a las explicaciones dadas sobre el experimento de Arago (Faraday) del imán y el disco de cobre? ¿Cómo?

- ¿Pasa cada punto del conductor en este caso por puntos de diferente fuerza magnética?

- ¿Cómo se generan entonces las corrientes en el disco?

- ¿Se podnia postular la existencia de algo" que se extiende fuera del imán e interactúa con el disco generando así las corrientes? ¿Si es así, qué cosa seria?

- ¿Se inducirá corriente con un sólo polo del imán?

\section{Experimento 4: Corrientes en función de la posición relativa de imán y disco}

Se coloca un disco de cobre o aluminio (con punto de apoyo) sobre una superficie horizontal y en un plano paralelo a éste, a unos $5 \mathrm{~cm}$ de altura, se suspende un imán de forma que pueda ser rotado. Al rotar el imán, el disco gira (Figura 5).

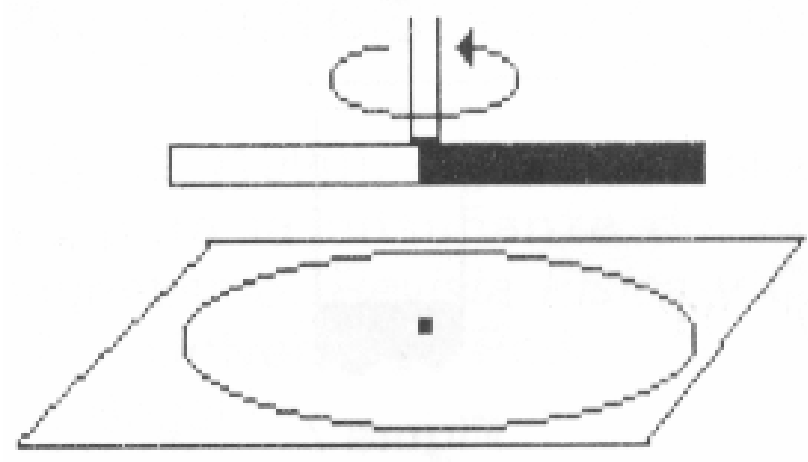

Figura 5

Ahora el imán se coloca vertical y se hace rotar (con el galvanómetro intentamos percibir corrientes) (Figura 6).

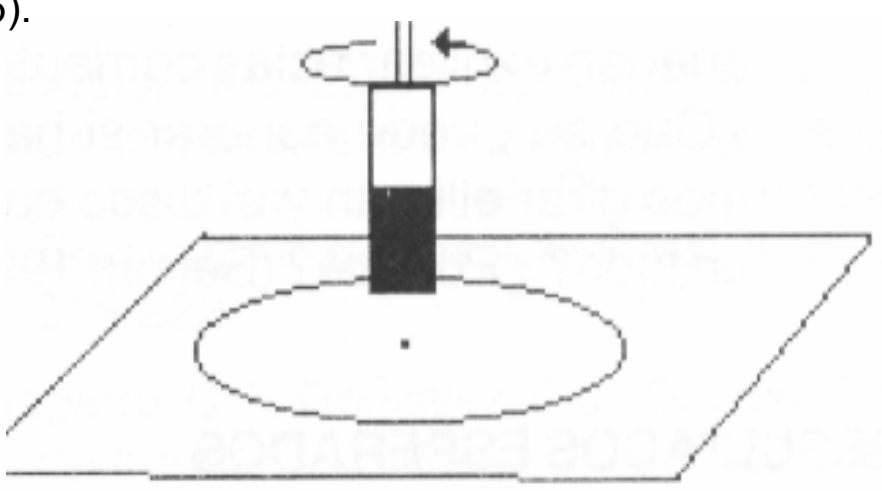

\section{Figura 6}




\section{En este caso el disco ya no rota.}

- ¿Porqué?

- ¿Qué diferencia hay con el caso anterior?

- ¿Debería inducirse alguna corriente en el disco? ¿Por qué?

- ¿Si era algo lo que interactuaba en el disco, por qué ahora ya no?

Ahora, se invierte la situación. Se deja el imán vertical fijo y sobre éste, se hace rotar el disco previniendo que no toque al imán; nuevamente con el galvanómetro intentamos percibir corrientes (Figura 7).

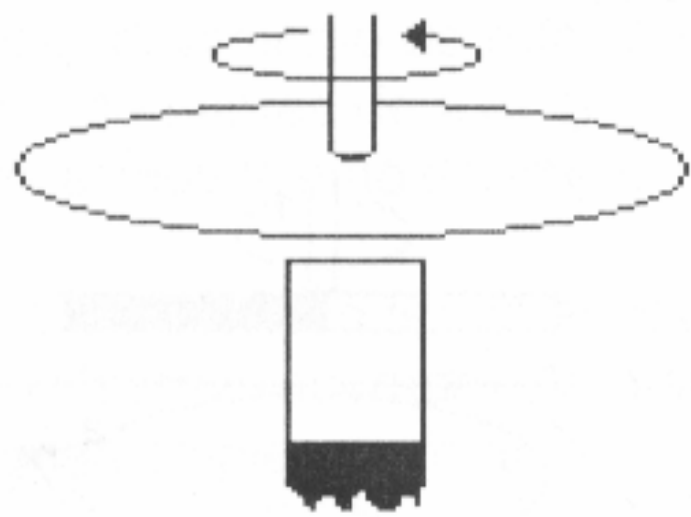

Figura 7

- ¿Al rotar el disco, pasa por puntos de diferente fuerza magnética?

- ¿Por qué ahora se induce corriente y no en el caso anterior?

- ¿Podría Ampére explicar este hecho? ¿Cómo?

- ¿Bajo qué nueva perspectiva se pueden explicar estas corrientes?

- ¿Qué se puede esperar si hacemos girar el imán y el disco como untado? ¿Porqué? (Beltrán 1996).

\section{RESULTADOS ESPERADOS}

Hasta aquí, se espera, que el estudiante haya comprendido ante todo la necesidad de un cambio de explicación y haya construido un peldaño más de conocimiento acerca de la evolución de la teoría electromagnética.

Las preguntas que se plantearon como ejemplo son opcionales, no necesariamente se tienen que hacer en ese orden, esto dependerá de cada maestro. A lo que sí debemos aspirar, si optamos por este tipo de aprendizaje, es que con la experiencia, dichas preguntas sean cada vez más concretas, sólidas y con buen grado de profundidad, que apunten a su vez hacia objetivos también concretos y que una vez inducido el estudiante en el proceso, sea él mismo quien se genere sus preguntas, su marco de reflexión (Driver, 1988).

Sobra decir entonces que el mismo papel del maestro cambia radicalmente, de ser quien da el conocimiento y las explicaciones a quien orienta y sugiere la búsqueda de información para procurar que alrededor del fenómeno se logre hacer un análisis lo más 
completo posible. Así, él también tiene que ser un investigador, estar a la caza constante de fuentes que enriquezcan este tipo de trabajo, coordinar las discusiones y estar atento a preparar los experimentos necesarios, que permitan a su estudiante construir los siguientes peldaños.

Finalmente, se puede pensar en la posibilidad de extender este tipo de aprendizaje al resto de la Física y a otras ramas de la ciencia, para lo cual se requerirán maestros que estén dispuestos a cambiar su método, a investigar, a buscar hechos claves, a reproducirlos y a hacer del estudiante un constructor de su propio conocimiento.

\section{CONCLUSIONES}

Debemos tomar conciencia sobre nuestro método de enseñanza para la física y admitir que nuestro propio crecimiento como maestros se detiene al optar por el facilismo y al no vencer la pereza a dejar de ser un repetidor de textos y un dador' de conocimiento. Lo importante para un espíritu inquieto e inconforme con respecto a una metodología tradiciorial, es que pueda pasar de la crítica al intento de acciones decididas.

Frente a la formación de nuevos maestros, con mayor razón se justifica esta torna de conciencia, que debe además, involucrar la intención de introducir cambios más profundos en los programas y en las metodologías.

Nuestra inquietud con respecto a la enseñanza de La física, es que su objetivo principal no debería ser la reconstrucción de esquemas puramente matemáticos (Husserl, 1990) para emplearlos luego como herramienta en la solución de ejercicios y problemas hipotéticos. De esta forma, anulamos cualquier posible visión que se tenga de la ciencia o conducimos a concepciones falsas sobre la misma. Por otra parte, al proporcionar las explicaciones, creamos un sentido de dependencia que evita que el estudiante sea constructor de su conocimiento, que se involucre con profundidad en el mismo y que propicia la aceptación de las explicaciones como verdades absolutas últimas y acabadas.

El cambio de objetivo que se propone, es que el estudiante sea guiado hacia la comprensión y reflexión de las teorías y su validez, a través (le la investigación y análisis sobre la evolución de las mismas, trabajo que integre la mayor cantidad de aspectos posibles, como por ejemplo el histórico, el filosófico, el social, el epistemológico (Gagliardi 1988). Nuestra tesis es que esto se puede lograr alrededor de los experimentos. Desde esta perspectiva, el experimento cambia radicalmente su función de elemento recomprobatorio a elemento de reflexión para la construcción del conocimiento. Así, uno de los papeles del maestro será ahora, investigar y buscar los experimentos claves que cumplan esta función y dirigir adecuadamente su observación (Duschl 1995, Mellado y Carracedo 1993).

\section{REFERENCIAS BIBLIOGRAFICAS}

Bautista, G. (1990). Algunas consideraciones para la enseñanza del electromagnetismo. Revista Física y Cultura, 1(2) - Universidad Pedagógica Nacional. Bogotá.

Beltrán L M. (1996). Del estado electrotónico a las líneas de fuerza (experimentos pedagógicos). Tesis de Maestría en Docencia de La Física. Departamento de Física. Universidad Pedagógica Nacional. Bogotá. 
Berkson, W. (1981). Las teorías de los campos de fuerza desde Faraday hasta Einstein. Alianza Universidad . Madrid.

Colombo, de C. L. y Fontdevila, P. A. (1990). Concepciones previas en el aprendizaje significativo del electromagnetismo. Enseñanza de las Ciencias, 8(3), pp 215-222.

Chaparro, C. I., González, F. J., Orozco, J.C., Pedreros, [3. 1., Vallejo, J. 1. (1997). Introducción a la física de procesos desde una perspectiva fenomenológica. Educación y Pedagogía. Vol. IX No 18. pp 117-129 Universidad de Antioquia, Facultad de Educación. Colombia.

Donald, M. B. (1993). "La enseñanza constructivista" - University of Missouri, Columbia U.S.A.

Driver, R. (1988). Un enfoque constructivista para el desarrollo del currículo en ciencias. Enseñanza de las Ciencias, 6(2), pp 109-120.

Duschl, [3. A. (1995). Más allá del conocimiento: Los desafíos epistemológicos y sociales de la enseñanza mediante el cambio conceptual. Enseñanza de las Ciencias. 13(1)1995.

Faraday, M. (1994). Experimental Researches in Electricity. Great Books of the Western World 42. Quinta impresión.

Flórez, O. [3. (1994). Hacía una pedagogía del conocimiento. McGraw-Hill. Bogotá.

Furió, C. y Guisasola, J. (1997). Deficiencias epistemológicas en la enseñanza habitual de los conceptos de campo y potencial eléctrico. Enseñanza de las Ciencias, 15(2), pp 259-271.

Furió, C. y Guisasola, J. (1998). Dificultades de aprendizaje de los conceptos de carga y de campo eléctrico en los estudiantes de bachillerato y universidad. Enseñanza de las Ciencias, 16(1), pp 131-146.

Furió, M. C. y Gil P. D. (1989). La didáctica de las ciencias en la formación inicial del profesorado: una orientación y un programa teóricamente fundamentados. Enseñanza de las Ciencias, 7(3)1989, 257-265.

Gagliardi, [3. (1988). Cómo utilizar la historia de las ciencias en la enseñanza de las ciencias. Enseñanza de las Ciencias, 6(3), pp 291-296.

Garrét, R. M. (1998). Resolución de problemas y creatividad: implicaciones para el currículo de ciencias. Enseñanza de las Ciencias, 6(3), pp 224-230.

Gil P. D. (1991).. ¿Qué hemos de saber y saber hacer los profesores de ciencias? Enseñanza de las Ciencias, 9(1)1991, pp 69-77.

Gil, P. D. (1983). Tres paradigmas básicos en la enseñanza de las ciencias. Enseñanza de las Ciencias, pp 26-33. 
Gómez, M. Y. (1998). El experimento de Oersted y la teoría electromagnética de Ampére (un análisis con intencionalidad pedagógica). Trabajo de grado. Universidad Pedagógica Nacional, departamento de Física. Bogotá.

Hammer, D. (1994). Epistemological beliefs in introductory physics. Cognition and Instruction, 12(2), pp 151-183.

Hilborn, [3. 0. (1998). (Am. J. Phys 56(11) enero 1988). Sobre el rediseño de cursos de física introductoria. Primeros niveles de universidad. Traducción de José González y Germán Bautista. Física y Cultura: Cuadernos sobre historia y enseñanza de las ciencias (4)1998. Universidad Pedagógica Nacional. Bogotá.

Hodson, D. (1988). Filosofía de la ciencia y educación científica. Traducción de Porlán García Ed Canal P. Sevilla, España.

Husserl, E. (1990) La crisis de las ciencias europeas la fenomenología transcendental. Una introducción a la filosofía fenomenológica. Ed. Crítica. Barcelona.

Mellado, V. y Carracedo, D. (1993). Contribuciones de la filosofía de la Ciencia a la didáctica de las ciencias. Enseñanza de las Ciencias 11(3)1993, 331-339.

Meneses, y. y Caballero, MC. (1995). Secuencia de enseñanza sobre el electromagnetismo. Enseñanza de las Ciencias, 13(1), pp 36-45.

Papert, S. (1982). Desafío a la mente. Computadoras y Educación. Ediciones Galápago. Bs.As.

Papert, S. (1995). La máquina de los niños. Paidós Ibérica SA. Barcelona.

Pozo, J. I., Pérez, M., Domínguez, J., Gómez, M. A. y Yolanda, P. (1994). La solución de problemas. Santillana de Ediciones. Madrid.

White, B. Y. (1993). Tinker Tools: causal models, conceptual change, and science education. Cognition and Instruction. Lawrence Erlbaum Associates, Inc. 10(1); pp: 1100. 